\title{
A Novel Method of Current Equalization in LED Strings Based on Simple Linear Circuit
}

\author{
Pedro S. Almeida ${ }^{1}$, João M. Jorge ${ }^{1}$, Cláudio R. B. S. Rodrigues ${ }^{1,2}$, Guilherme M. Soares ${ }^{1}$, \\ Danilo P. Pinto ${ }^{1}$ and Henrique A. C. Braga ${ }^{1}$ \\ ${ }^{1}$ NIMO - Modern Lighting Research Group - Federal University of Juiz de Fora (UFJF) \\ ${ }^{2}$ IFSEMG - Federal Institute of Education, Science and Technology of the Southeast of Minas Gerais \\ pedro.almeida@engenharia.ufjf.br; henrique.braga@ufjf.edu.br
}

\begin{abstract}
This paper focuses on the problem of driving parallel strings of LEDs while ensuring current equalization between them. This issue is addressed in terms of proposing a small and simple linear topology that can be used on a number of seriesassociated LEDs, working as a constant current source for each string. Such topology employs a four-component circuit, with low dependence on transistor parameters, ensuring easy repeatability and good current regulation. Mathematical description of the circuit and experimental results are provided.
\end{abstract}

Keywords - LEDs; LED string driving; current equalization; solid state lighting

\section{INTRODUCTION}

Solid-state lighting (SSL) has been recently finding its way on modern lighting systems, changing the perspectives of indoor as well as outdoor illumination. The advent of the socalled high-brightness power LEDs (HB-LEDs) to produce light is recent, since LEDs have been used for many years solely as indicators and luminous flags.

Due to high efficacy, long term estimations are that a $10 \%$ decrease on worldwide energy consumption could be attained by using LEDs, a $50 \%$ decrease on the electrical energy used for producing artificial lighting [1]. That, together with the white light and good color rendering index (CRI), makes LEDs a very attractive and environmentally friendly alternative as a light source.

But since HB-LEDs are essentially low power devices (usually 1 to $5 \mathrm{~W}$ ), an LED-luminaire (or anything equivalent of a lamp made out of LEDs) could not be constructed out of a single device. Thus, it is necessary to associate a number of LEDs in order to produce the desired light output.

There is a number of ways to associate LEDs in order to make a luminaire or a string module, each of them yielding advantages and disadvantages. Some will be analyzed in section II of this paper.

The main objective of this work is to propose a novel circuit to perform current balancing in series-parallel strings of LEDs. Experimental validation of the topology is provided for a two-string module of 32 power LEDs.

\section{ASSOCIATION OF LEDS}

The association of LEDs to make a string module raises concern on the current regulation of the individual devices or strings. Since light output and correlated color temperature are highly dependent on the forward current of an LED [2], there is a great deal of care to drive all the devices in a single string (or string module) under the same forward current.

Also, there is the possibility of an LED failure, under which different associations will respond in different manners. According to [3], LEDs tend to commonly fail as an open circuit under electrostatic discharge (ESD) and thermomechanical stress of the bond wires. But they can also fail as a short circuit, prevalently on-die, as a result of threading dislocations (formation of nanopipes on the substrate) or degraded passivation (defects on the insulating $\mathrm{SiO}_{2}$ layer).

A few possibilities for associating LEDs to form a string or luminaire module are shown in Fig. 1.

A pure series association of LEDs (Fig. 1-a), called an LED string, has the advantage of forcing all devices to share the same current, thus making any further current equalization unnecessary, so there's no current imbalance. On the other hand, large series strings could result in high string voltage. Plus, if a single LED turns out to fail as an open circuit, the whole string will be out, compromising the reliability of the module under this faulty condition.

A parallel association of strings forms a series-parallel LED module (Fig. 1-b). This reduces the impact of an open circuit LED failure, which would make only a string turn out, but creates a current imbalance issue. Since not all LEDs can be ensured to be identical, the voltage at each string will be slightly different, thus their currents at will also differ. Therefore, it might be necessary some sort of equalization circuit to balance the current on the individual strings.

A matrix module (Fig. 1-c) is made by connecting the individual LEDs in parallel and then connecting these parallel clusters in a series association.

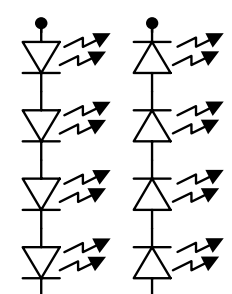

(a)

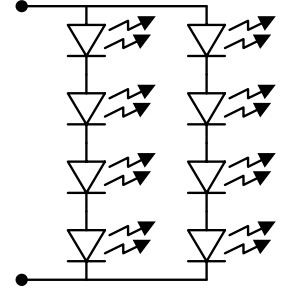

(b)

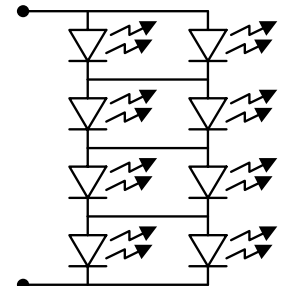

(c)
Fig. 1. Three possibilities of LED string modules: (a) series string, (b) series-parallel string module and (c) matrix module. 
The result is that every matrix row of the module shares the same voltage. Once more, current imbalance will take place, because LEDs intrinsically differ in forward voltage. But in this case, it would be necessary an equalization device for each LED, which happens to be unviable. One advantage is that an open circuit failure of an LED will result in only one device out, but if the failure turns out to be a short circuit, a whole parallel cluster will turn off due to the voltage drop to zero.

\section{EQUALIZATION TECHNIQUES FOR LED STRINGS}

The series-parallel association of LEDs turns out to be an alternative that can be, at the same time, more reliable under faulty conditions (such as open circuit failure) than the pure series strings, and more easy for current balancing than the matrix module. Therefore, the only problem to be solved is how to equalize the currents at the parallel-associated strings.

A number of circuits and techniques have been proposed for equalizing the current in LED strings, ranging from fairly simple to more intricate topologies. Most of these circuits assume a constant voltage bus or a current source, from where the LEDs and equalization circuits are fed. This bus can be provided by an adequate switching regulator, as in Fig. 2.

Some of the techniques use monolithic linear regulators, such as LM317, plus a pass transistor to provide the strings with regulated, equalized currents [4]. This provides a fairly simple circuit, but the efficiency can be quite low for the higher currents required by power HB-LEDs, because the regulator IC requires a minimum voltage drop from input to output for the circuit to work.

Another linear alternative are MOSFET-based current mirrors [5]. A reference branch MOSFET, which has its gate connected to other branches of LED strings, forces the mirroring of the reference current on these strings. This circuit only works satisfactorily if the MOSFETs are high-gain and identical (or very alike), which is a virtually unattainable condition for discrete power MOSFETs. Plus, the reference branch drains current without any useful output, and the circuit uses as many MOSFETs as LED strings.

A more intricate circuit is presented in [6], and uses a linear regulator along with a feedback loop to control the switching pre-regulator, in order to adapt the output voltage to keep the efficiency as high as possible.

These three linear equalization circuits are shown in Fig. 3, Fig. 4 and Fig. 5, respectively.

There are also the switching alternatives for current equalization. For example, one presented in [7] uses an inverter based on the current-fed parallel-resonant push-pull topology. The LEDs are connected in anti-parallel at the output of the inverter, two-by-two, series-connected to a current balancing capacitor. The LEDs are fed by half-wave rectified high-frequency quasi-sinusoidal current and zerovoltage switching can be attained, thus also higher efficiency.

Some other more intricate switching circuits are proposed by [10] and [11], and a hybrid switching-magnetic solution is proposed in [12]. The switching solutions are more varied and complex by definition, and will not be further explored in this work.

Linear circuits, though commonly more inefficient, are usually easier to construct and cost-effective, requiring less parts and being sufficiently reliable for mass-manufacturing luminaires. Thus, a novel linear alternative is proposed in the next section.

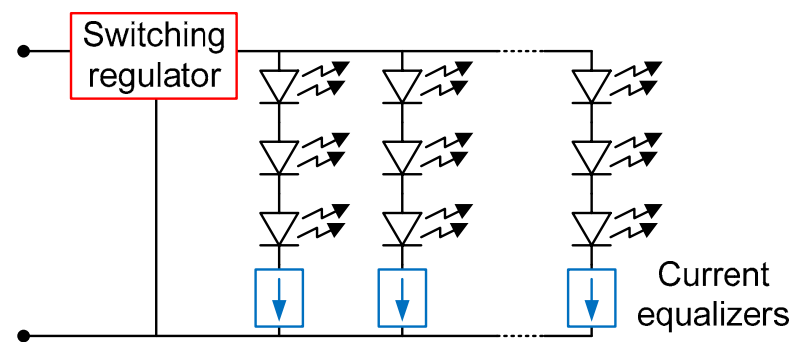

Fig. 2. Basic scheme of a series-parallel modular HB-LED luminaire.

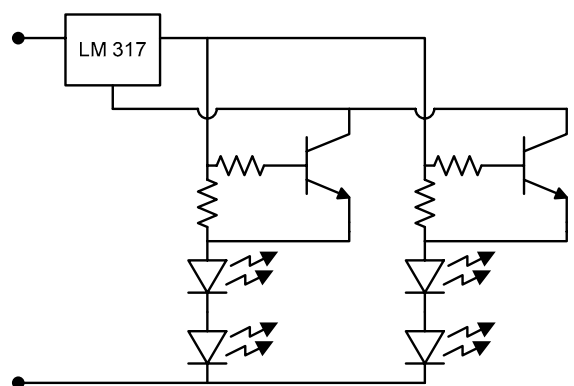

Fig. 3. Linear current equalizer using monolithic regulator IC.

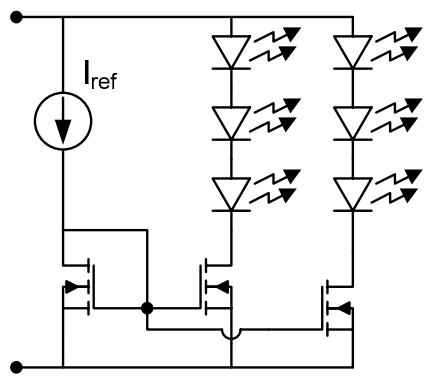

Fig. 4. Linear current equalizer using current mirrors.

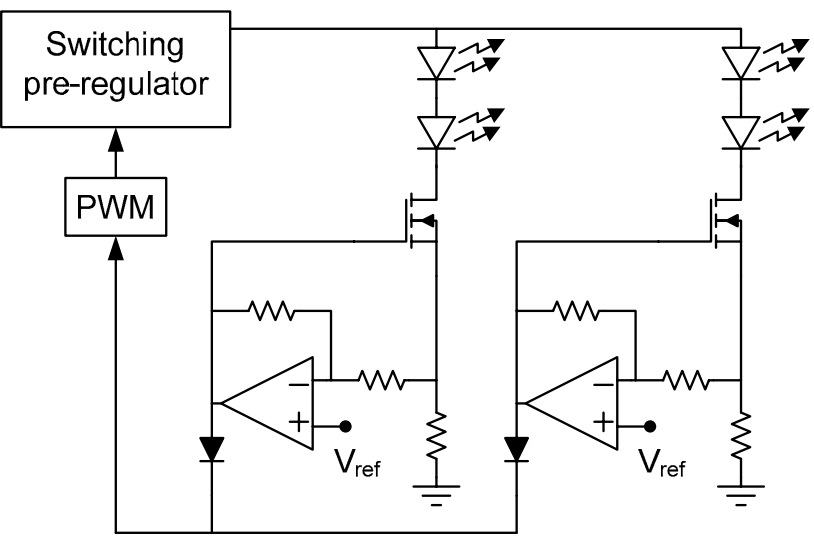

Fig. 5. Linear current equalizer using adaptive drive voltage. 


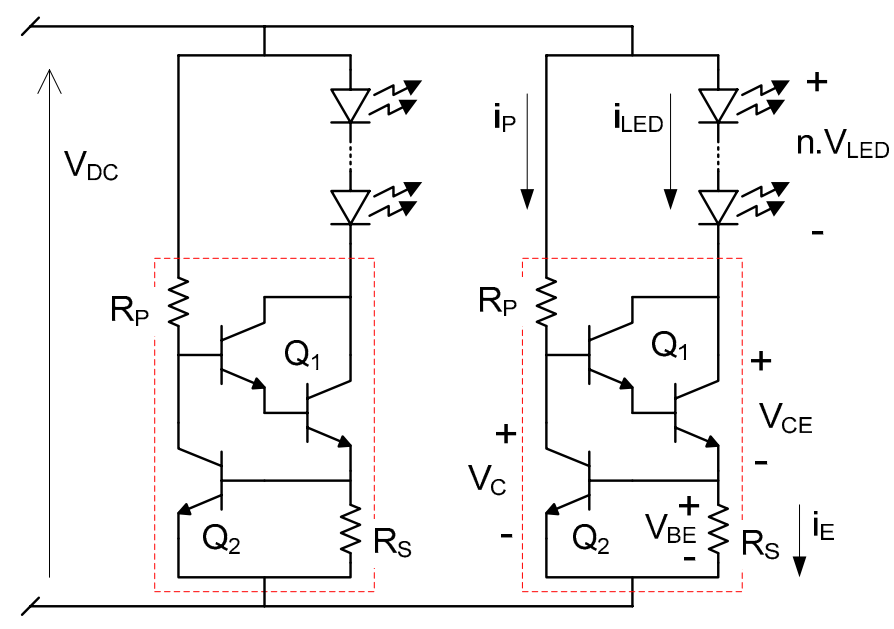

Fig. 6. Proposed current equalizer circuit, here shown for two LED strings.

\section{Proposed CIRCUIT AND ANALYSIS}

The circuit proposed of a linear current equalizer for LED strings is shown in Fig. 6. This circuit uses only two low power resistors, one signal bipolar transistor and one monolithic Darlington-pair transistor, in order to act as a constant current source for each string of LEDs.

The strings are assumed to be fed by a constant voltage (e.g., from a switching regulator) that is slightly higher than the string voltage itself. One of the main advantages is that this voltage difference can be extremely low, circa 1 volt.

The circuit works by forcing the current that feeds the LEDs on the collector of the transistor $\mathrm{Q}_{1}$ to be almost equal to the constant current at its emitter. $\mathrm{Q}_{2}$ is used to provide a constant voltage reference for the current-defining resistor $R_{S}$, as low as possible in order to avoid great losses on the circuit.

It is good to stress that a complementary PNP version of the circuit could also be implemented, using a similar analysis to the described below.

Assuming that both transistors $\mathrm{Q}_{1}$ and $\mathrm{Q}_{2}$ are operated at active-mode and their current gains being given by $\beta_{1}$ and $\beta_{2}$, respectively, (1) follows.

$$
i_{L E D}=i_{E} \frac{b_{1}}{b_{1}+1}
$$

Since $\beta_{1}$ has a very large value, due to the Darlington-pair configuration, and $\mathrm{Q}_{2}$ is a high-gain signal transistor with low base current, the approximation (2) can be used.

$$
i_{L E D} \gg i_{E}=\frac{V_{B E}}{R_{S}}
$$

Thus, the LED string current is a function solely of two constants: the base-emitter voltage of $\mathrm{Q}_{2}$ and the value of the resistor $\mathrm{R}_{\mathrm{S}}$. $\mathrm{V}_{\mathrm{BE}}$ is only dependent on the transistor $\mathrm{Q}_{2}$ chosen.

The polarizing current $i_{P}$ is of small value, enough only to produce the voltage reference at $\mathrm{Q}_{2}$. The voltage across the
LED string is given by the estimated voltage of one device times the number of series associated LEDs ( $\left.n . V_{\text {LED }}\right)$. Thus, the theoretical efficiency of the circuit can be obtained as the ratio between output and input powers, approximated by (3).

$$
h=\frac{P_{L E D}}{P_{i n}}=\frac{n \cdot V_{L E D} \cdot i_{L E D}}{\left(i_{P}+i_{L E D}\right) V_{D C}} \gg \frac{n \cdot V_{L E D}}{V_{D C}}
$$

\section{DESIGN GUIDELINES AND CONSIDERATIONS}

Equation (3) shows that the efficiency of the circuit is directly proportional to the number of series associated LEDs in a single string. Therefore, this could be used as a design guideline: using as long as possible strings and as low as possible input voltage for attaining high efficiencies. This idea could be also implemented at the control loop of the switching pre-regulator assumed to be present before the equalizers, playing the role of a maximum efficiency point tracker.

For a practical design to work, it should be ensured that the transistor $\mathrm{Q}_{1}$ is working at active-mode. In order for that to happen, its collector-emitter voltage $V_{C E}$ should be kept at a higher value than the saturation voltage of the same device, $\mathrm{V}_{\mathrm{CE} \text {,sat }}$. That imposes a bottom limit for the input voltage, given by (4).

$$
V_{D C} \neq n \cdot V_{L E D}+V_{C E, s a t}+V_{B E}
$$

Maximum efficiency of the design will be attained at the lower limit imposed by (4), thus defining $\mathrm{V}_{\mathrm{DC}}$.

The voltage at the collector of transistor $\mathrm{Q} 2\left(\mathrm{~V}_{\mathrm{C}}\right)$ can be used to define the polarizing resistor $R_{P}$, leading to (5).

$$
R_{P}=\frac{V_{D C}-V_{C}}{i_{P}} @ \frac{V_{D C}-3 \cdot V_{B E}}{i_{P}}
$$

The polarizing current $i_{P}$ could be chosen to assume a value between $100 \mu \mathrm{A}$ and $1 \mathrm{~mA}$ for a small-signal transistor.

Finally, the current defining resistor $\mathrm{R}_{\mathrm{S}}$ could be chosen by modifying (2) to (6).

$$
R_{S}=\frac{V_{B E}}{i_{L E D}}
$$

Its power rating is always a constant value, dependent on LED and transistor parameters together, and given by (7) .

$$
R_{S} \cdot i_{L E D}^{2}=V_{B E} \cdot i_{L E D}
$$

The power rating of the $R_{S}$ resistor is less than half a watt for the common power LEDs that operate on currents below $700 \mathrm{~mA}$. This means that a small signal resistor can be used.

These design guidelines can be easily applied to obtain a working prototype in order to test the circuit's performance. 


\section{PROTOTYPE DESIGN AND EXPERIMENTAL RESUlTS}

A prototype for a two-string LED module is proposed for design validation. The strings are composed of 16 seriesconnected OSRAM LUW W5PM - Golden DRAGON Oval Plus LEDs. These LEDs have a typical light output of 106 lumens each at a drive current of $350 \mathrm{~mA}$, while having a typical forward voltage of $3.2 \mathrm{~V}$ at the same current [8].

This gives a two-string module of 32 LEDs, with a nominal light output of almost 3400 lumens, which is closely equivalent to a $35 \mathrm{~W}$ metal-halide lamp. Each string will have an approximate voltage of $51.2 \mathrm{~V}$ and the whole module is expected to demand something close to $36 \mathrm{~W}$.

For the current equalizer, the transistors chosen were a small-signal BC337 NPN for the polarizing branch and a TIP110 NPN Darlington for the LED string branch. These devices are high-gain transistors, suitable for this design.

The transistors' electrical parameters of interest were experimentally evaluated. The $\mathrm{V}_{\mathrm{BE}}$ voltage of $\mathrm{Q}_{2}$ transistor was found to be $0.6 \mathrm{~V}$ when forward-biased at $i_{\mathrm{P}}=500 \mu \mathrm{A}$. As for the saturation voltage of $\mathrm{Q}_{1}, \mathrm{~V}_{\mathrm{CE} \text {,sat }}$, it was measured $0.7 \mathrm{~V}$ at $350 \mathrm{~mA}$. The parameters of the design are shown in Table I.

From (4), it is possible to derive the minimum bus voltage for feeding the LED module: $\mathrm{V}_{\mathrm{DC}} \neq 52.5 \mathrm{~V}$. Using this value, the resistance of $R_{P}$ is calculated from (5), being $R_{P} @ 100 \mathrm{k} \Omega$.

The current defining resistor is calculated from (6) as being $R_{S}=1.7 \Omega$. These prototype components are summarized in Table II.

A photograph of the LED module plus the built-in prototype is shown in Fig. 7. The experimental results of each string are presented together in Fig. 8, while the input voltage and current are shown in Fig. 9. The relevant experimental data obtained are compiled in Table III. Module efficiency (strings plus equalizer) was measured $93.6 \%$, at full power.

TABLE I. DESIGN PARAMETERS

\begin{tabular}{ccc} 
Symbol & Value & Parameter \\
\hline \hline $\mathrm{i}_{\mathrm{LED}}$ & $350 \mathrm{~mA}$ & LED string current \\
$\mathrm{V}_{\mathrm{LED}}$ & $3.2 \mathrm{~V}$ & LED forward voltage drop \\
$\mathrm{n}$ & 16 & number of LEDs in a string \\
$\mathrm{i}_{\mathrm{P}}$ & $500 \mu \mathrm{A}$ & polarizing current \\
$\mathrm{V}_{\mathrm{BE}}$ & $0.6 \mathrm{~V}$ & base-emitter voltage of Q2 \\
$\mathrm{V}_{\mathrm{CE}, \text { sat }}$ & $0.7 \mathrm{~V}$ & saturation voltage of Q1
\end{tabular}

TABLE II. PROTOTYPE COMPONENTS

\begin{tabular}{ccc} 
Symbol & Value & Parameter \\
\hline \hline $\mathrm{R}_{\mathrm{S}}$ & $1.7 \Omega$ & current defining resistor \\
$\mathrm{R}_{\mathrm{P}}$ & $100 \mathrm{k} \Omega$ & polarizing branch resistor \\
$\mathrm{V}_{\mathrm{DC}}$ & $52.5 \mathrm{~V}$ & bus input voltage
\end{tabular}

TABLE III. EXPERIMENTAL RESULTS

\begin{tabular}{cccc} 
Measurement point & Current & Voltage & Power \\
\hline \hline String 1 & $350.1 \mathrm{~mA}$ & $49.72 \mathrm{~V}$ & $17.4 \mathrm{~W}$ \\
String 2 & $344.7 \mathrm{~mA}$ & $49.84 \mathrm{~V}$ & $17.2 \mathrm{~W}$ \\
\hline Input & $705.3 \mathrm{~mA}$ & $52.4 \mathrm{~V}$ & $36.96 \mathrm{~W}$
\end{tabular}

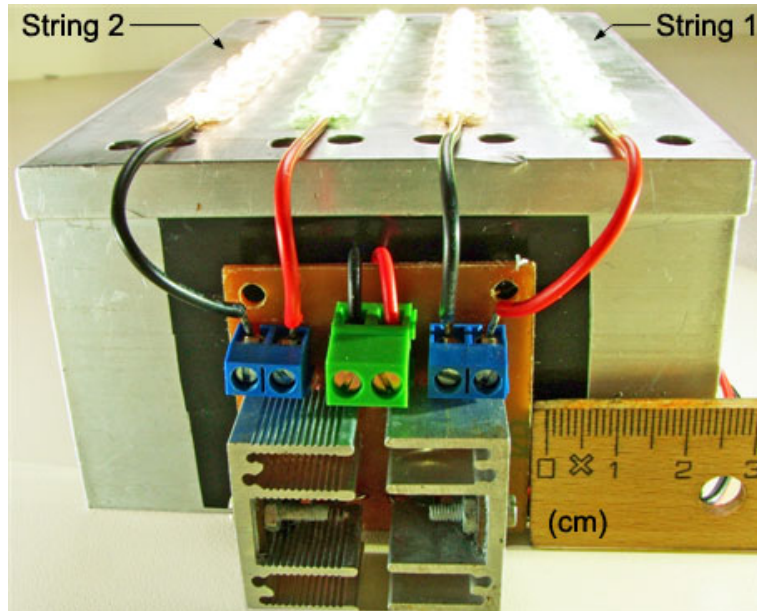

Fig. 7. Current equalizer circuit on two-string $36 \mathrm{~W}$ LED module.

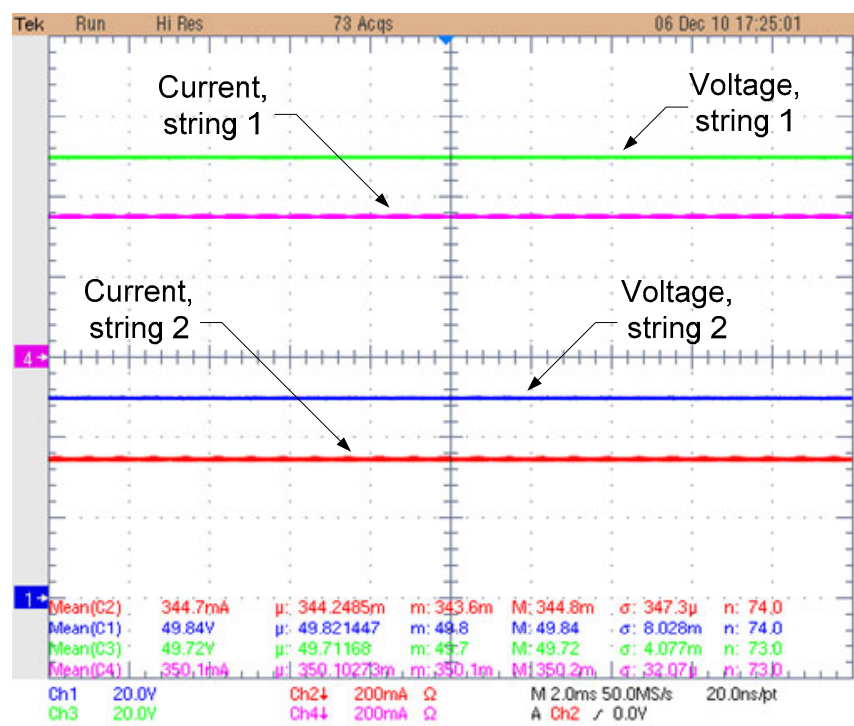

Fig. 8. Current and voltage at both LED strings (200 mA/div; $20 \mathrm{~V} / \mathrm{div})$.

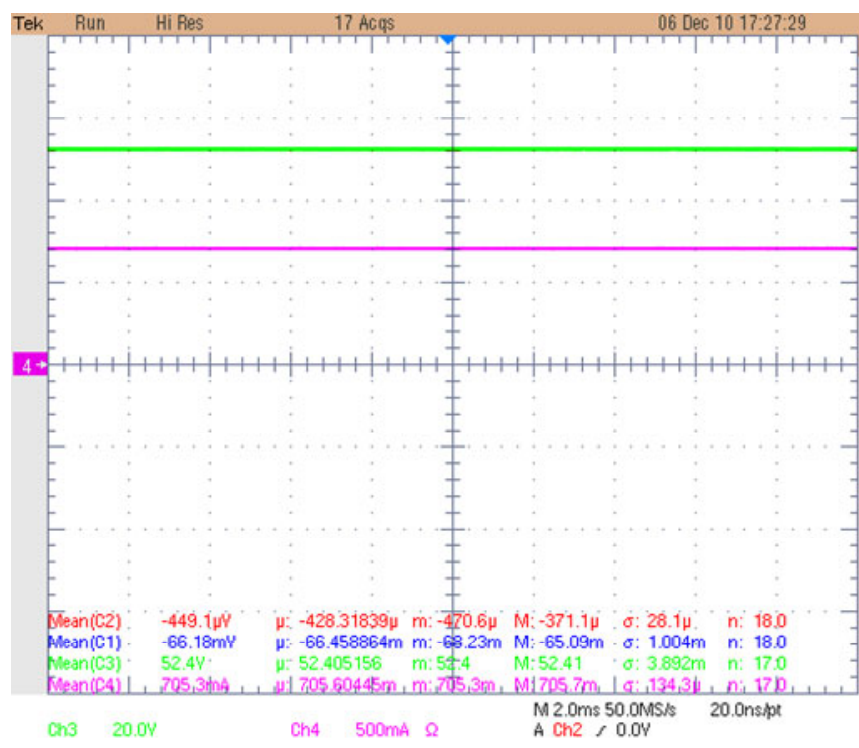

Fig. 9. Current (bottom trace) and voltage (upper trace) at input of LED module $(500 \mathrm{~mA} /$ div; $20 \mathrm{~V} /$ div $)$. 


\section{TEST UNDER FAULTY CONDITIONS}

For testing the behavior of the circuit under LED failure, were considered both scenarios - open circuit and short circuit failures.

If one LED fails open, his whole string will turn off, but the other strings will remain current-regulated by the other independent equalizing circuits, and no damage will be caused to them or the LEDs. The module would remain working, although the light output would decrease by a factor of $1 / \mathrm{k}$, where $\mathrm{k}$ is the number of strings being fed, for each string that is put out (e.g., one string out of two means a $50 \%$ reduction; two strings out of three means a $66 \%$ luminosity reduction).

On the other hand, a short circuit failure would apply an overvoltage on the devices, in case there was no current equalizing circuit. But the proposed scheme is intended to compensate this kind of failure, by maintaining the current regulated and equalized throughout the strings.

Since shorts are the most common failure mode of LEDs [9] and also have a more significant impact for the equalizing circuit proposed, a failure test was conducted, with different numbers of LEDs in different strings failing short. The current at each string was measured in all cases, to ensure that equalization would still take place even under abnormal conditions. The results of these tests are presented at Table IV.

The small difference between values in a same string suggests that the measured discrepancies are only ordinary fluctuations on current. In this case, the circuit is able to sustain equalization under LED short circuit failure satisfactorily.

\section{CONCLUSION}

This paper presented a simple and compact circuit applied to the current equalization on parallel strings of seriesassociated LEDs. A brief analysis of its working principle was carried out and a design guideline was developed.

Current equalization can have a great impact on the light output of LED modules or luminaires, since small changes in device voltage can yield large changes in current, implying in color and luminous flux deviation, or yet uneven aging of the devices.

Thus, having an equalizing technique or circuit may be mandatory on larger luminaires, in order to keep reliability and quality of illumination.

Low cost solutions, such as small but sufficiently efficient linear equalizers, may turn out to be a viable way out. The circuit here presented had a good experimental validation, while relying on only 4 ordinary electronic components.

TABLE IV. LED FAILURE TEST - SHORT CIRCUIT FAILURE

\begin{tabular}{cccc}
$\begin{array}{c}\text { Number of } \\
\text { failed devices }\end{array}$ & $\begin{array}{c}\text { String of } \\
\text { failure }\end{array}$ & $\begin{array}{c}\text { Current at } \\
\text { string 1 }\end{array}$ & $\begin{array}{c}\text { Current at } \\
\text { string 2 }\end{array}$ \\
\hline \hline 1 LED & String 1 & $349.1 \mathrm{~mA}$ & $343.9 \mathrm{~mA}$ \\
3 LEDs & String 1 & $348.1 \mathrm{~mA}$ & $344.3 \mathrm{~mA}$ \\
\hline 1 LED & String 2 & $349.5 \mathrm{~mA}$ & $345.5 \mathrm{~mA}$ \\
3 LEDs & String 2 & $348.7 \mathrm{~mA}$ & $344.4 \mathrm{~mA}$
\end{tabular}

Still, it achieved an efficiency of almost 94\%, while the current deviation between the two LED strings fed through the equalization circuit was only $1.5 \%$. These experimental results should validate both the circuit and the analysis performed, but a brief qualitative comparison between the two mainstream equalizing philosophies is drawn in Table $\mathrm{V}$ in order to point each one's limitations and generalized characteristics. As shown, a number of technically "positive" features may qualify small linear solutions as good alternatives for current balancing in strings of LEDs, mainly cost, low electromagnetic (EMI) emissions and simplicity.

Low parts count yields reliability and cost-effectiveness, while efficiency-related problems could be countered with adequate regulation of input voltage. The evidence for good current balancing is compelling enough to justify the use of such simple approach presented in this work.

Also, the behavior of the circuit under LED short circuit failure was investigated. Current equalization was maintained under all tested failures, with the maximum current deviation between the two strings being $1.6 \%$, under abnormal conditions.

It is clear, however, that efficiency will drop when one or more LEDs fail short, due to the intrinsic linear nature of the circuit. Nevertheless, the current on a string containing a short-failed LED will maintain its nominal value, in order not to damage any more devices. Thus, the current equalizer plays its roll on maintaining the reliability of the whole module.

A proposal for a complete luminaire is to employ a switching pre-regulator before the strings plus equalizers, and implementing a control loop that is able to guarantee the minimum required voltage for equalization to take place, by measuring the string voltage and making the bus voltage only higher enough than the string voltage itself. Thus, the highest efficiency possible could be attained for the whole set, as if in a maximum efficiency point tracking control scheme.

Future studies may be proposed to investigate the global electrical and photometrical impact of such an equalization circuit on large LED modules, as in public lighting luminaires and other outdoor and indoor solid-state lighting applications.

Furthermore, electrical transients, temperature drift and LED aging effects on the equalizer could be assessed in future work, as well as using more paralleled strings to test the circuit's response in larger modules.

TABLE V. COMPARISON BETweEn Equalizing TeCHNiQues

\begin{tabular}{ccc} 
& $\begin{array}{c}\text { Switching Equalizing } \\
\text { Solutions }\end{array}$ & $\begin{array}{c}\text { Linear Equalizing } \\
\text { Solutions }\end{array}$ \\
\hline \hline Operating frequency & High & Low (DC) \\
Current balancing & Complexity-dependent & Fair to good \\
EMI & Moderate to high & Low to none \\
Cost & Moderate to high & Low \\
Parts count & Medium to high & Low \\
Reliability & Complexity-dependent & High \\
Efficiency & High & Input voltage-dependent \\
Heating & Moderate to low & Moderate to high
\end{tabular}




\section{ACKNOWLEDGMENT}

Authors would like to thank the financial and technical support of Eletrobras, CAPES, FAPEMIG and CNPq, as well as OSRAM Opto Semiconductors for ceding the LEDs used on the prototype of the luminaire module under which the equalizing circuit was tested.

\section{REFERENCES}

[1] J. Y. Tsao, "Solid state lighting: lamps, chips and materials for tomorrow," IEEE Circuits \& Devices Magazine, vol. 20, no.3, pp. 2837, 2004.

[2] G. Carraro, "Solving high-voltage off-line HB-LED constant current control-circuit issues," IEEE Applied Power Electronics Conference and Exposition (APEC), pp. 1316-1318, 2007.

[3] J. Arnold, "When the lights go out: LED failure modes and mechanisms," White Paper, Dfr Solutions, College Park, Margland.

[4] On Semiconductor, "AND8109-D - LED constant current source scheme: theory of operation," Application Note, 2003.
[5] C. Correa, J. Garcia, C. Barriquello, A. Schittler, D. Camponogara and R. N. Prado "Aplicação de espelhos de corrente no acionamento de LEDs de potência," Congresso Brasileiro de Automática (CBA), 2008.

[6] Y. Hu and M. M. Jovanovic, "A novel LED driver with adaptive drive voltage," IEEE Applied Power Electronics Conference and Exposition (APEC), pp. 565-571, 2008.

[7] S. M. Baddela and D. S. Zinger, "Parallel connected LEDs operated at high frequency to improve current sharing," IEEE Industry Applications Conference / 39th IAS Annual Meeting, vol. 3, pp. 1677-1681, 2004.

[8] Osram Opto Semiconductors, "LUW W5PM Golden Dragon Oval Plus," Datasheet, 2010.

[9] Amtex Electronics, "Application Note: 1 - LED Power Supplies, Common LED Configurations," Application Note, 2009.

[10] C. Chiu and K. Chen, "A High Accuracy Current-Balanced Control Technique for LED Backlight," IEEE Power Electronics Specialists Conference (PESC), pp. 4202-4206, 2008.

[11] Texas Instruments, "UCC28810EVM-003 - 110-W Multiple String LED Driver with Universal Line Input and PFC," User's Guide, 2009.

[12] K. I. Hwu and S. Chou, "A simple current-balancing converter for LED lighting," IEEE Applied Power Electronics Conference and Exposition (APEC), pp. 587-590, 2009. 